\title{
Link Scheduling in a Single Broadcast Domain Underwater Networks
}

\author{
Pai-Han Huang, Ying Chen and Bhaskar Krishnamachari \\ Ming Hsieh Department of Electrical Engineering \\ Viterbi School of Engineering \\ University of Southern California \\ Los Angeles, California 90089 \\ Email: \{paihanhu, chen2, bkrishna\}@usc.edu
}

\author{
Anil Kumar \\ Virginia Bio-Informatics Institute \\ and Department of Computer Science \\ Virginia Tech Blacksburg, VA 24060, USA \\ akumar@vbi.vt.edu
}

\begin{abstract}
Due to the high propagation latency and high power consumption of acoustic communications, scheduling techniques designed for terrestrial radio-based systems, may not be suitable for underwater acoustic sensor networks (UWASN). In this paper, we consider how to time schedule each link in a single broadcast domain. We show that, unlike its terrestrial RF counterpart, this problem is NP-complete, and the hard-to-approximate ratio is presented. Due to the intractability and inflexibility of centralized scheduling policies, and the high communication energy overhead of reservation-based strategies, we further investigate the performance of an ALOHA-like access scheme, which is distributed, randomized and requires no topology knowledge. According to our analysis, although the random scheduling policy that picks transmission times uniformly in a given interval is throughputoptimal for terrestrial radio-based systems, it performs poorly in underwater acoustic networks. We thereby seek for the throughput-optimal, distributed random policy by solving a nonlinear optimization problem. We present an extensive comparison between this policy and the uniform one, with respect to different packet lengths, scheduling length, and network density. We show that the optimal solution offers substantial improvements in throughput, particularly for long packets.
\end{abstract}

\section{INTRODUCTION}

Because radio waves decay rapidly in water, acoustic communication is the most popular choice for underwater sensor networks. Different from radio, acoustic communication has its own characteristics, such as high communication energy consumption, low bandwidth, long delay, high error rate, distance-dependent bandwidth, etc. These features make the scheduling for UWASN fundamentally different from radiobased communication systems. For example, because the propagation speed of acoustic waves is 5 order slower than radio, carrier-sensing and reservation based protocols will lead to low utilization in underwater acoustic networks, while this is general not the case for terrestrial RF based systems. In addition, a standard modem takes $50 \mathrm{~W}$ to transmit and couples watts to listen and receive data [1]. It is prohibitively expensive to transmit frequently in such a power-scarce environment. Other issues, such as simplex communication hardware constraints, low transmission rate, small communication bandwidth etc., all make the scheduling problem under this environment challenging [2][3][1].

The first key contribution of this work is that we consider the algorithmic complexity and hardness of the UWASN scheduling problem. We show that this problem is NP-complete, and it is impossible to approximate within $\left(\frac{n}{2}\right)^{1-\epsilon}, \forall \epsilon \quad>$ 0 (where $\mathrm{n}$ is the number of nodes in the network), unless $N P=Z P P^{1}$.

Besides being computationally hard, centralized scheduling has other limitations. For example, in order to apply a centralized scheduling protocol, several conditions, e.g. complete knowledge of network topology, are required, thus incurring high communication overhead. Whenever changes of topology take place, or nodes lose synchronization, centralized algorithms require to repeat scheduling decision making processes, thus becoming inflexible. Hence, a distributed scheduling scheme is preferable in this scenario. There are different flavors of distributed scheduling protocols. Among them, carrier-sensing cannot function well for UWASN due to the long propagation delay. On the other hand, reservationbased schemes require extensive packet exchange to determine transmission eligibility, thus also suffering from high communication overhead. Due to these unwanted properties, we are interested in a light energy overhead, low deployment effort, and versatile to network dynamics MAC. The answer to our needs is therefore a Distributed, Randomized and Topology-unaware (DRT) scheduling strategy. Given that optimal scheduling is hard even to approximate well, and DRT solutions are preferable, we focus on analyzing and optimizing a distributed random access protocol for UWASN. Our second key contribution is to show that unlike in terrestrial RF networks, uniform access is not optimal. We formulate and solve a novel non-linear optimization problem to find the optimal access time distribution, which is demonstrated to give enormous improvements in throughput. For brevity, we refer to a DRT scheduler that picks the transmission times uniformly from a given period as a uniform DRT scheduler. A scheduler, which is obtained by using nonlinear programming and utilizes DRT strategy, is referred to as a optimal DRT scheduler. We present the achievable throughput by using

\footnotetext{
${ }^{1} \mathrm{ZPP}$, Zero-error Probabilistic Polynomial time, is a complexity class of problems, such that there exists a probabilistic Turing machine, which always returns correct YES or NO answers in polynomial time on average [4].
} 
DRT strategy, and compare the performance of uniform DRT scheduler and optimal DRT scheduler. Based on our findings, optimal DRT scheduler prevails in low density and long packet length setting. Compared with uniform DRT scheduler, the throughput improvement ratio keeps increasing while packet length goes up.

This paper is organized as follows. We demonstrate the NPcompleteness and hard-to-approximate ratio in Section III. The analysis of uniform DRT scheduler is presented in Section IV, and the optimal DRT scheduler is derived in Section V. We characterize the features of optimal DRT scheduler, and compare its performance with uniform DRT scheduler in Section VI Our findings and future directions are summarized in Section VII.

\section{RELATED WORK}

Due to the features of acoustic communication, some existing work focuses on designing new MAC protocols. Syed et al. [5] study the performance of traditional slotted and nonslotted ALOHA performance for underwater networks and show that they can have the same throughput due to variations in latency. They then propose a guard-band scheme to improve the throughput. This scheme is mathematically analyzed and optimized by Ahn et al. in [6]. Syed et al. in [5] have further proposed a reservation based scheme called T-Lohi. Molins et al. use ideas similar to slotted-CSMA/CA to design a reservation based MAC protocol, Slotted-FAMA[7]. Because Slotted-FAMA uses RTS/CTS mechanism to avoid collision, this protocol suffers from low channel utilization and high handshake communication energy overhead. Nguyen et al. [8] and Casari et al. [9] compare the existing MAC protocols, and review the pros and cons of various underwater medium access techniques under different network setting. Preisig et al. [10] focus on acoustic channel property descriptions and provide propagation phenomena that can influence network performance in a quantitative way. Other than these directions, Badia et al. [11] propose a cross-layer optimization framework, which utilizes integer programming, that can determine link scheduling and packet routing. To our knowledge, there are no prior results on the computational complexity of scheduling in underwater networks.

The paper that's closest to our work in spirit is the work by Ahn et al. [6] in that both focus on an analysis of randomized access in underwater networks. However, while that work focuses on the improvement of throughput for a single receiver using guard-bands, our focus is on maximizing the sumthroughput of multiple links in the same broadcast domain by optimizing the access time distribution. This is more challenging because in underwater networks, the same transmission may appear as interference at significantly different times at different receivers.

\section{Algorithmic CompleXity}

We consider a time-slotted, single carrier frequency and single communication power setting system. Each node we considered is located in the same broadcast domain, i.e. each

transmission can be overheard by all nodes. There are two kinds of receptions in the viewpoint of a receiver; one is intended transmission and the other one is interference. An intended transmission is the data sent by the corresponding sender of this receiver, and an interference is data sent by nodes other than its corresponding sender. We assume a transmission has high enough power to corrupt an intended transmission, even it has propagated the longest distance within the deployed area. Every transmission can only take place at the beginning of a time slot, and spans exactly one time slot. We assume no coding protection for all transmissions. Therefore, an intended transmission fails, if and only if a collision happens. By collision, we refer to the condition that an intended transmission is corrupted by interferences. Take Figure 1(a) for example, if transmitter 1 and 2 transmit in slot 1 and 10, respectively, then both transmissions arrive receiver 2 at slot 11 , thus causing collision.

Our interest is, "given a set of transmitter-receiver pairs $P$, and pair-wise delay between any two nodes, which satisfy a metric ${ }^{2}$, is it possible to schedule all transmissions within a schedule period with length $K$, such that no collision will corrupt intended communications?" We call this problem "Metric Underwater Scheduling", and claim that it is NPcomplete.

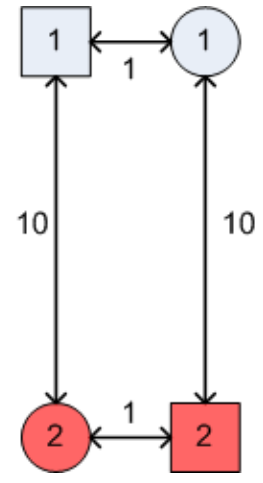

(a)

10
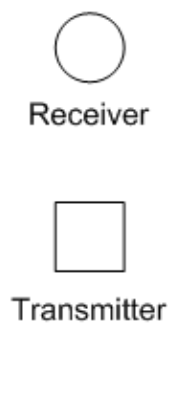

Fig. 1. Examples of network topology.

Theorem 1: Metric Underwater Scheduling is NP-complete.

Proof:

A schedule with length $K$ is a certificate of the Metric Underwater Scheduling. We could check this schedule, in polynomial time, whether every transmitter is scheduled within $K$ and no intended communication is corrupted by interference. Therefore, Metric Underwater Scheduling is in NP.

To prove NP-hardness, we do the reduction from K-Coloring [12]. Consider an arbitrary instance with graph $G=(V, E)$ of K-Coloring, we build a corresponding graph $H=\left(V^{\prime}, E^{\prime}\right)$ as follows. For every node $v \in V$, we build a gadget consisting of two nodes $v_{1}^{\prime} \in V^{\prime}$ and $v_{2}^{\prime} \in V^{\prime}$, such that $v_{1}^{\prime}$ and $v_{2}^{\prime}$ can be

\footnotetext{
${ }^{2}$ A valid metric satisfies the following properties: (1) non-negativity , (2) identity of indiscernible, (3) symmetry, and (4) triangle inequality.
} 
considered as a transmitter and a receiver in Metric Underwater Scheduling, respectively. Note that, if the highest degree of $G$ is $\triangle, K$ must be no less than $\triangle$ to be feasible. Connect every transmitter and receiver pair in $H$ with an edge, which has length $a>K$. The length of an edge in $H$ represents the propagation delay between the pair of nodes connected by this edge. For every edge $(u, v) \in E$, we add two edges, $\left(u_{1}^{\prime}, v_{2}^{\prime}\right)$ and $\left(v_{1}^{\prime}, u_{2}^{\prime}\right)$, with length $a$ in $H$. If two nodes, say $x$ and $y$, are not connected by an edge in $G$, then we add two edges, $\left(x_{1}^{\prime}, y_{2}^{\prime}\right)$ and $\left(y_{1}^{\prime}, x_{2}^{\prime}\right)$, with length $2 a$ in $H$. In this construction, the exhaustive combination of edges which form a triangle can be listed as: $\{a, a, a\},\{a, 2 a, a\},\{2 a, 2 a, a\}$. Obviously, they satisfy triangular inequality. In addition, distances are nonnegative and symmetry, thus, the construction of $H$ satisfies a metric.

We first prove that, if vertexes of $G$ are $K$ colorable, then we can construct a solution of Metric Underwater Scheduling as follows: for each color used in K-Coloring, we map it to a unique time slot within $K$. If we denote the color of node $v \in V$ as $c(v)$, then we assign its corresponding time slot, $t(v)$, to the transmitter $v_{1}^{\prime} \in H$. Because $G$ is $K$ colorable, two vertexes, say $u$ and $v$ in $G$, which are joint by a edge, say $(u, v)$ in $G$, can not have the same color. In other words, transmitter $u_{1}^{\prime}$ and $v_{1}^{\prime}$ in $H$ can not transmit at the same time slot because of interference. On the other hand, if two vertexes, say $x$ and $y$ in $G$, are not connected by an edge, then they can be assigned either the same color , i.e., $t(x)=t(y)$, or different, i.e., $t(x) \neq t(y)$, and both corresponding assignments are feasible in $H$. First, consider $t(x)=t(y)$. Since the packets of both $x_{1}^{\prime}$ and $y_{1}^{\prime}$ will arrive at their intended receivers and other's intended receivers at time $t(x)+a$ and $t(x)+2 a$, respectively, no collision occurs under this schedule. If $t(x) \neq t(y)$, then transmission of $x_{1}^{\prime}$ will arrive $y_{2}^{\prime}$ at time $(t(x)+2 a)>2 a$. However, $y_{2}^{\prime}$ already receives its packet at time $(t(y)+a) \leq$ $(2 a-1)$. Similarly, we can verify that $y_{1}^{\prime}$ will not interfere with $x_{2}^{\prime}$ intended communication. Thus K-colorable implies a feasible solution of Metric Metric Underwater Scheduling.

Conversely, we will prove that if Metric Underwater Scheduling has a satisfying solution, then $G$ is also Kcolorable. For a transmission schedule, say $t(u)$ for transmitter $u_{1}^{\prime}$, we map it to a unique color within the $K$ choices. If a receiver, say $v_{2}^{\prime}$, is connected with another transmitter, say $u_{1}^{\prime}$, with a length $a$ edge in $H$, then we know $u_{1}^{\prime}$ and $v_{1}^{\prime}$ will interfere with each other, thus will not be scheduled in the same slot. By the above mapping, $u$ and $v$ in $G$ are also colored in different colors. However, we know that $(u, v) \in E$. Therefore, this color assignment is feasible in $G$. If a receiver, say $y_{2}^{\prime}$, is connected with another transmitter, say $x_{1}^{\prime}$, with a length $2 a$ edge in $H$, then we know there does not exist an edge $(x, y) \in G$. Therefore, there is no direct conflict on color choices between node $x$ and $y$ in $G$. Thus, we construct a viable K-Coloring solution from Metric Underwater Scheduling.

Further, we can have the following conclusion:

Theorem 2: If we denote the number of nodes in Metric

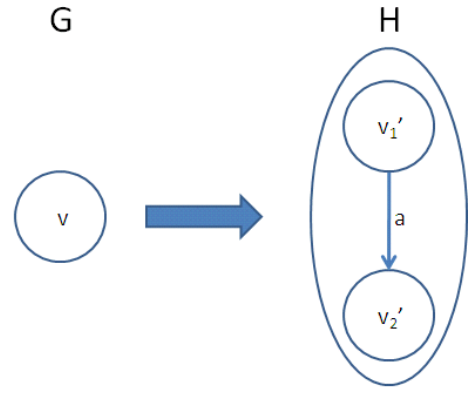

(a) Construction of a gadget

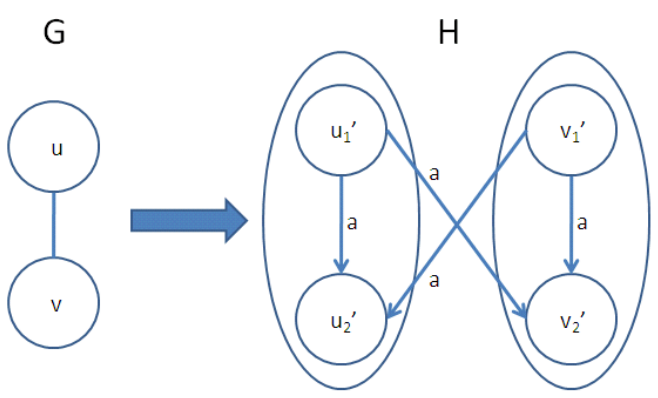

(b) Mapping an edge of $G$ to $H$

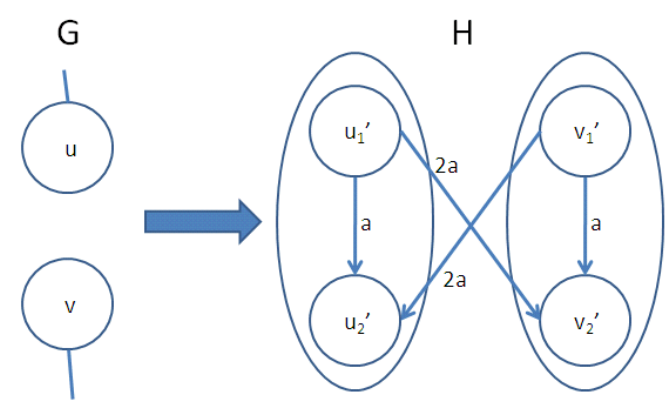

(c) Mapping an edge of $G$ to $H$

Fig. 2. Construction of gadgets.

Underwater Scheduling as $n$, then it is not possible to approximate Metric Underwater Scheduling within $\left(\frac{n}{2}\right)^{1-\epsilon}, \forall \epsilon>0$, unless NP $=$ ZPP.

Proof: Suppose the original K-coloring instance has $K$ nodes, then the corresponding Metric Underwater Scheduling instance has $2 K$ nodes. According to the above proof, we notice that the number of minimum chromatic number equals to the minimum schedule length. If we have $\frac{n}{2}^{1-\epsilon}=K^{1-\epsilon}, \forall \epsilon>$ 0 approximation for Metric Underwater Scheduling, then it implies that we can approximate Minimum Chromatic Number within $K^{1-\epsilon}, \forall \epsilon>0$, which contradicts to the conclusion proposed by Feige and Kilian [4].

The most challenging issue of dealing with underwater scheduling problem is the non-negligible propagation delay: the same transmission may be considered as interference by different nodes at significantly different times. Therefore, the interference pattern not just depends on the time the packet has been sent, but also relates to the network topology. In short- 
ranged terrestrial RF networks, because propagation delay is negligible, once a packet is transmitted, it arrives every node in the same broadcast domain immediately. Therefore, the minimum number of time slots we need to time schedule every link once within a given period, equals to the number of links in the same broadcast domain.

On the contrary, UWASN allows concurrent transmission without incurring collision due to the fact of high propagation latency. Therefore, it is possible to schedule multiple, say $X$, transmission pairs with scheduling length less than $X$ slots. An example of this scenario is depicted in Figure 1(a). However, when a network has high density, it is more likely that we may need a scheduling period with length larger than the number of transmission pairs. An example of this situation is plotted in Figure 1(b).

\section{Performance AnAlysis of Uniform DRT SCHEDULER}

As mentioned in the introduction, because of several undesired features of centralized schedulers, reservation-based protocols and carrier-sensing protocols, we are interested in the performance that DRT strategy can achieve in UWASN. Due to its throughput superiority and simplicity for terrestrial RF networks, uniform DRT scheduler, which picks the transmission times uniformly from a given period, is analyzed in this section as a starting point to help us understand the applicability and limitations of DRT strategy.

\section{A. System Modeling}

For the sake of generality, we use "normalized distance unit" and "normalized time unit" in the following context. For example, if a network of interest has dimension $1000 \mathrm{~m} \times 1000 \mathrm{~m}$, then we will denote the area as $1 \times 1$. In addition, " 1 unit of normalized time" in this example represents the actual time, in unit of second, for the sound wave to travel $1000 \mathrm{~m}$.

Consider the following settings. In a $1 \times 1$ square area, a network is independently, uniformly and randomly deployed with $2 N$ nodes. Exactly $N$ of them are transmitters, and the remaining nodes are receivers. Each transmitter is uniquely and exclusively coupled with a receiver, and can transmit one packet in one schedule period. In other words, there are exactly $N$ links located in this broadcast domain. The length of a schedule period is $K$ units of normalized time, and each transmitter independently chooses an instance within this period to start transmitting a packet according to a continuous uniform distribution. Moreover, every packet takes exactly $P$ units of normalized time to transmit, and a transmission fails if and only if collision takes place. The assumptions about transmission power, coding and transmission bands are the same as in Section III. The symbols we use are listed in Table I. We are interested in the probability of a successful transmission, system throughput (defined later), and per user throughput using this random scheduler.

\section{B. Theoretical Analysis}

First, the PDF (Probability Density Function) of random variable $Z_{i j}$ (the distance between node $i$ and $j$ ) is described by Square Line Picking problem [13]. By applying elementary probability techniques, we can find the exact PDF of random variable $A_{i j}$ (the first bit, transmitted by node $i$, arrival time at node $j$ ). Since every node is independently deployed, and transmission times are also independently determined, we abbreviate $Z_{i j}$ and $A_{i j}$ to $Z$ and $A$, respectively. However, due to the tedious expressions of PDF of $A$, we describe a more insightful approximation in this paper, and refer interested readers to [14].

A good approximation of $A$ can be stated as follows: when $K \leq 1.3$, PDF of $A$ can be approximated as a normal distribution; when $K>>1.3$, PDF of $A$ can be approximated as a uniform distribution within the range $(0, K+\sqrt{2})$. Examples of the distribution of $A$ for small $K$ and large $K$ are plotted in Figure 3(a) and 3(b), respectively. When $K>1.3$, the PDF of $A$ always has a flat region as plotted in Figure 3(b), and the width of this region becomes wider, as $K$ increases.

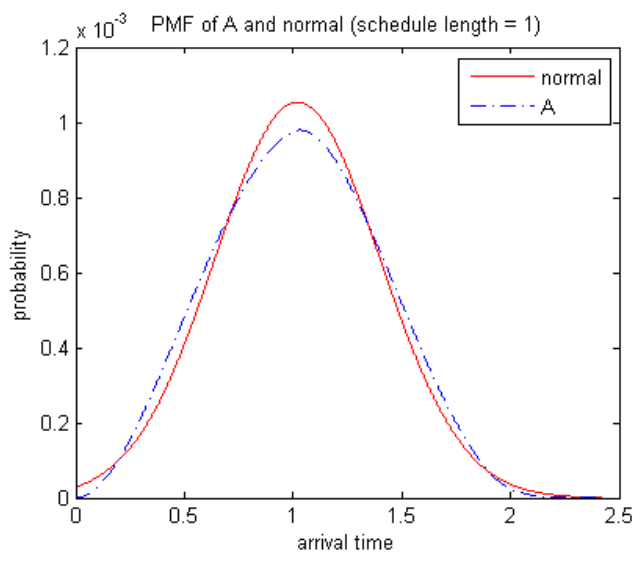

(a) A vs. normal (small $\mathrm{K}$ value)

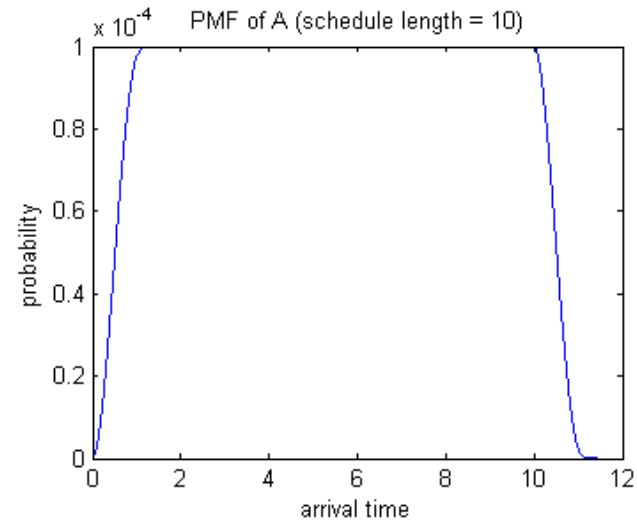

(b) Distribution of $\mathrm{A}$ (large $\mathrm{K}$ value)

Fig. 3. Example distribution plots of $\mathrm{A}$ for small $\mathrm{K}$ and large $\mathrm{K}$.

Note that, although the scenario we discuss so far is for "one" scheduling period only, independently iterating this random access process can be a viable MAC protocol. However, in order to prevent transmissions taking place in prior schedule 
TABLE I

DEFINITION OF SYMBOLS

\begin{tabular}{|c||l||l|}
\hline$K$ & the schedule length $\quad P$ & the packet length \\
\hline$N$ & num of transmission pairs in the same broadcast domain \\
\hline$A_{i j}$ & a RV representing the 1 st bit transmitted by node $i$, arriving time at node $j$ \\
\hline$Z_{i j}$ & a RV representing the Euclidean distance between node $i$ and $j$ \\
\hline
\end{tabular}

periods from interfering with consequent ones, using "guard time" between two consecutive schedule periods is necessary. In our case, the longest propagation delay is $\sqrt{2}$ unit of normalized time and the packet length is $P$, thus $\sqrt{2}+P$ is proper for our purpose. Because decisions of transmission time are made independently across different users and different schedule periods, the above iterating scheme can be considered as a renewal process. Therefore, the throughput can be calculated as:

throughput $=$ average amount of delivered data in one schedule period length of one schedule period

Because of independency, the numerator of Equation 1 can be computed as:

(average amount of delivered data in one schedule period) $=$ $N \times$ (probability a transmission succeeds) $\times($ packet length $)$

If we denote the PDF and CDF (Cumulative Density Function) of $A$ as $f_{A}$ and $F_{A}$, respectively, then a transmission will be corrupted by interferences with probability:

$$
\operatorname{Pr}(\text { collision })=\int_{0}^{K} f_{A}(j) \int_{\max (0, j-P)}^{\min (K, j+P)} f_{A}(k) d k d j
$$

Therefore, the probability a transmission succeeds is $1-$ $\operatorname{Pr}$ (collision). For the sake of space restriction, the exact expressions of the probability that a packet can be successfully delivered are listed in our technical report [14]. We will present plots showing the throughput of this uniform scheme with respect to various parameters in the next section, after describing the optimal DRT scheduler.

\section{Observations}

One of the main difference between the PDF of $A$ plotted in Figure 3(a) and 3(b) is the shape: while $K$ is small, the PDF has an obvious peak, whereas the other one has flat top. In Figure 3(a), around $80 \%$ of transmissions start arriving a receiver within time 0.516 to 1.528 , which represents only $41 \%$ of total possible arrival period. On the contrary, in Figure 3(b), around $80 \%$ of transmissions arrive within time 1.522 to 9.521 , which is over $70 \%$ of total possible arrival period. Therefore, when schedule length $K$ is small, using uniform DRT scheduler will lead to high collision probability, due to the concentration of transmission arrival pattern. The throughput of using uniform DRT scheduler is even more problematic when longer packets are used. On the other hand, although the first bit arrival time for the case of long schedule length distributes more evenly across whole possible arrival period, as we will see in Section VI, uniform DRT scheduler still suffers from long transmission packets, thus leading to low throughput.

\section{Optimal DRT ScheduleR}

In light of our findings, we seek for the throughput-optimal scheduler, which uses DRT strategy, in this section. Because of the consideration of computation complexity, we approximate PDF with PMF (Probability Mass Function), which is derived from discretizing PDF, in this section.

\section{A. Problem Formulation}

System throughput is determined by Equation 1 and 2. If packet length $P$, schedule length $K$, and number of transmission pairs $N$ are constants, then maximizing throughput is equivalent to minimizing collision probability. We denote the transmission time generated by optimal DRT scheduler and its Gorresponding first bit arrival time as $T^{*}$ and $A^{*}$, respectively. Because DRT strategy does not utilize the knowledge of network topology, $f_{A^{*}}(a)$, PMF of $A^{*}$, can be calculated as:

$$
f_{A^{*}}(a)=\sum_{i} f_{T^{*}}(i) f_{Z}(a-i)
$$

The collision probability becomes:

$$
\operatorname{Pr}(\text { collision })=\sum_{j}\left(f_{A^{*}}(j) \sum_{|j-k|<P} f_{A^{*}}(k)\right)
$$

Therefore, our goal can be achieved by solving the following optimization problem:

$$
\begin{array}{ll}
\text { minimize: } & \sum_{j}\left(f_{A^{*}}(j) \sum_{|j-k|<P} f_{A^{*}}(k)\right) \\
\text { subject to : } & f_{T^{*}}(t) \geq 0, \forall t \\
& \sum_{t} f_{T^{*}}(t)=1
\end{array}
$$

\section{B. Characteristics of Optimal DRT Scheduler}

We use Matlab optimization toolbox to solve the above problem, and 1 unit of time is divided into 100 segments in our PMF computation. In general, we have the following observations:

- When $K<1$, the optimal DRT scheduler only picks the first and the last available transmission instances. In this case, if $K>>P$, then these two instances have equal probability to transmit; if $K \sim P$, then the last available instance has higher probability. An example PMF of optimal DRT scheduler is plotted in Figure 4(a).

- When $K \geq 1$, the optimal DRT scheduler may use some instances in the middle of the schedule period. If $K>>$ $P$, then the PMF of optimal DRT scheduler may look 
like the circle/red set of points in Figure 4(b); if $K>P$, then the PMF of optimal DRT scheduler may look like the plus/blue set of points in Figure 4(b); if $K \sim P$, then the PMF of optimal DRT scheduler has the shape of the circle/red set of points in Figure 4(a).

- When $K>>1$ and $K>>P$, then the uniform DRT scheduler is close to optimal.

- As plotted in Figure 5, compared with uniform DRT scheduler, optimal DRT scheduler tends to distribute the transmission arrival time more evenly, so as to avoid the traffic concentration issue.

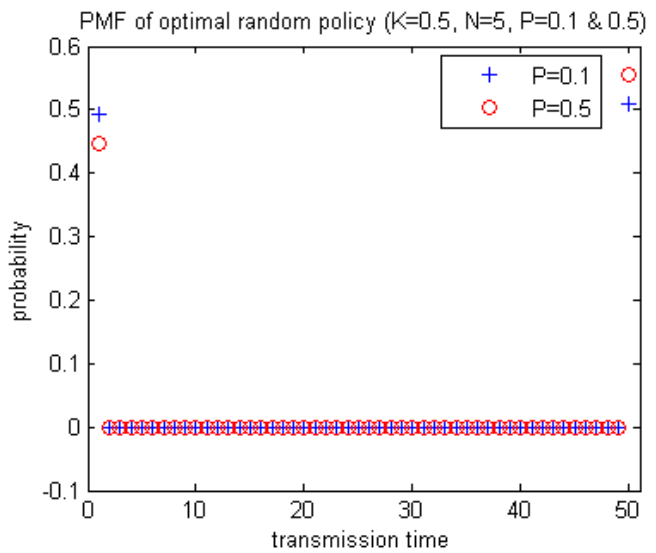

(a) small $\mathrm{K}$ value

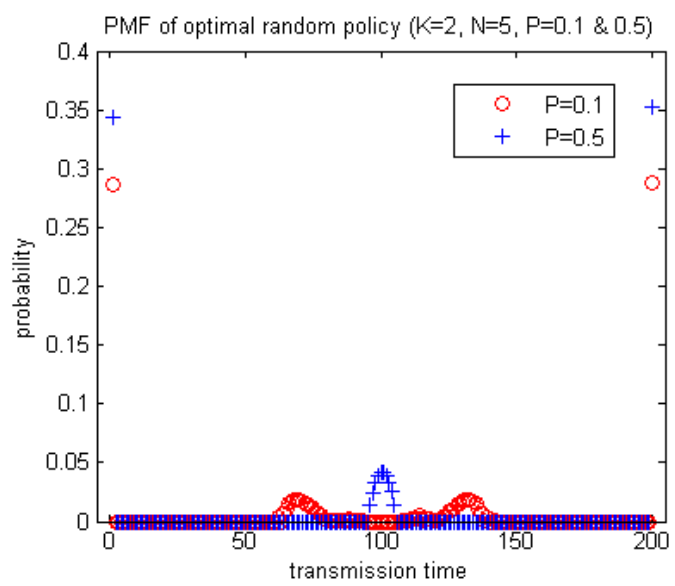

(b) large $\mathrm{K}$ value

Fig. 4. Example PMF plot of optimal random policy for small $\mathrm{K}$ and large $\mathrm{K}$.

\section{Discussion}

In Figure 6(a), we vary $K$ and set $N=2$ and $P=$ $\{0.5,1.5\}$. Because there are only two pairs, contention is light, thus leading to low collision probability when packet is short. When $P=0.5$, the improvement of using optimal DRT scheduler, compared with uniform DRT scheduler, is $22 \%$. However, when longer packet $P=1.5$ is used, collision probability raises significantly for uniform DRT scheduler. On the contrary, collision probability of optimal DRT scheduler degrades more gracefully, thus benefiting from using longer

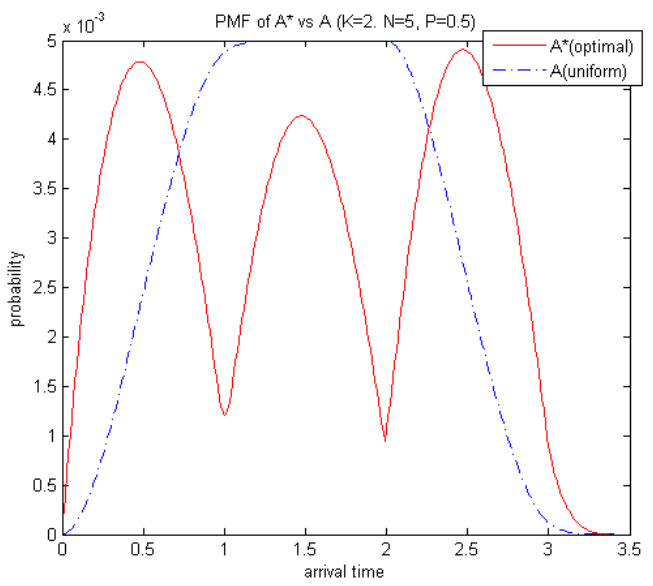

Fig. 5. Distribution of first bit arrival time: $\mathrm{A}^{*}$ (optimal) vs. A(uniform)

packets. When $P=1.5$, optimal DRT scheduler has around 4 times of throughput than uniform DRT scheduler, and this ratio keeps increasing when $P$ goes up as plotted in Figure 7 . More importantly, the maximum throughput of optimal DRT scheduler is even higher than $P=0.5$. In general, optimal DRT scheduler prevails under long packet settings.

The major difference between Figure 6(b) and 6(a) is the increase of network density from $N=2$ to $N=7$. When $N=7$, because contention is high, both schedulers suffer in this setting. Although the improvement ratio of using optimal DRT scheduler is even higher (around 9 times) when $P=1$, the achievable throughput is still low and may not be an ideal operational choice.

In Figure 6(c), we vary $N$ and set $K=0.5$ and $P=$ $\{0.5,1\}$. For both schedulers, the throughput optimal density is $N=2$, and experience low throughput in this short schedule length $K=0.5$ setting. While we increase $K$ to 2 in Figure 6(d), both schedulers still prefer low network density. Therefore, it is not suitable to deploy DRT strategybased schedulers in heavy contention environments.

In Figure 6(e), we vary $P$ and set $K=0.5$ and $N=\{2,6\}$. While $N=2$, both schedulers can benefit from using longer packet. As contention raises ( $N$ increases to 6$)$, no one can take advantage of long packet, and need to use the shortest packet to maximize throughput. The throughput gain of using optimal DRT scheduler also shrinks, as the network density goes up.

To understand how the throughput optimal packet length varies with schedule length $K$, we compare both schedulers in Figure 6(f). It is noticeable that both schedulers choose to use longer packets, while schedule length increases. In addition, the throughput gain of optimal DRT scheduler, compared with uniform DRT scheduler, also increases with schedule length.

We summarize our findings as follows:

- When schedule length and density are fixed, the throughput improvement ratio of using optimal DRT scheduler, compared with uniform DRT scheduler, increases as packet length goes up as plotted in Figure 7. 


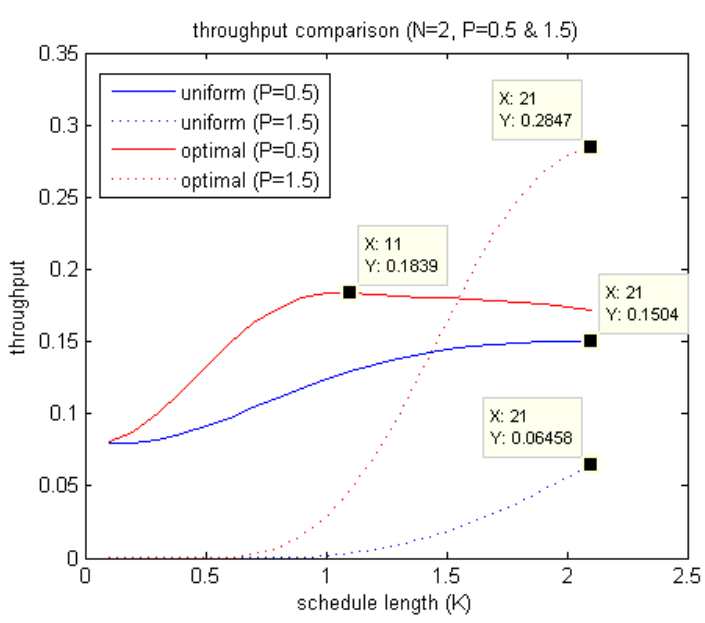

(a)

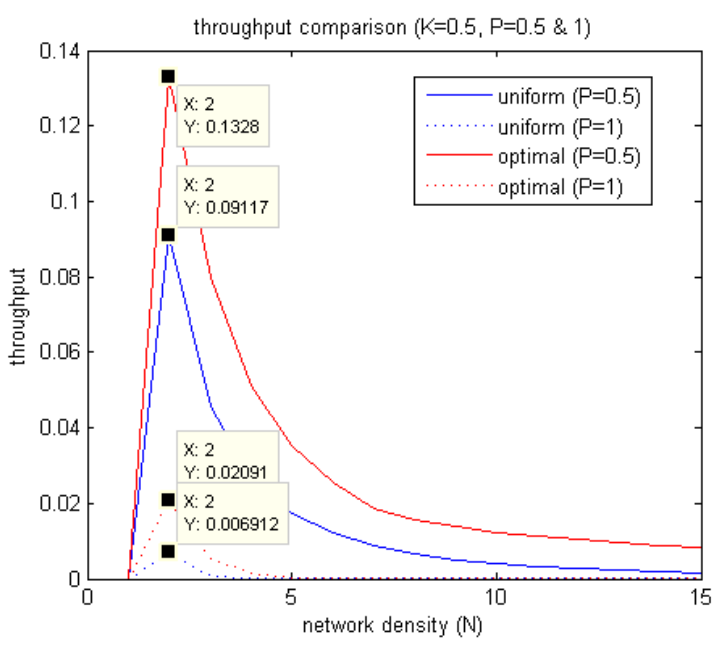

(c)

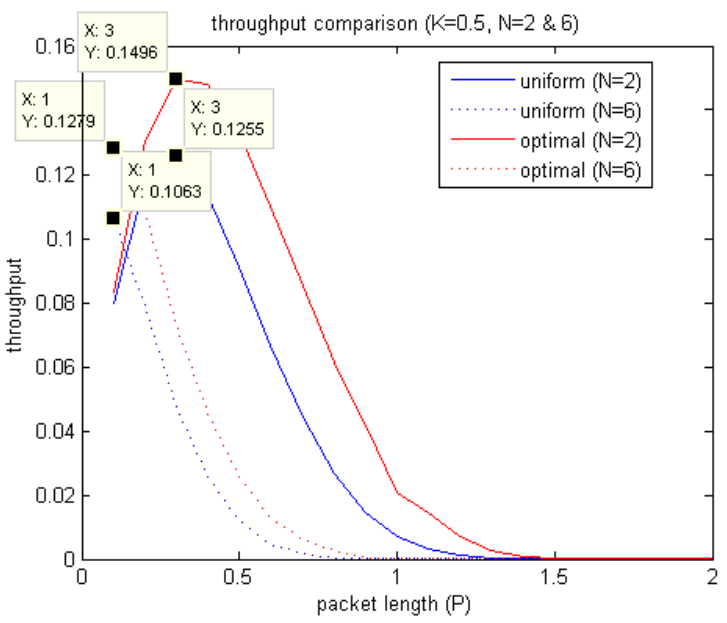

(e)

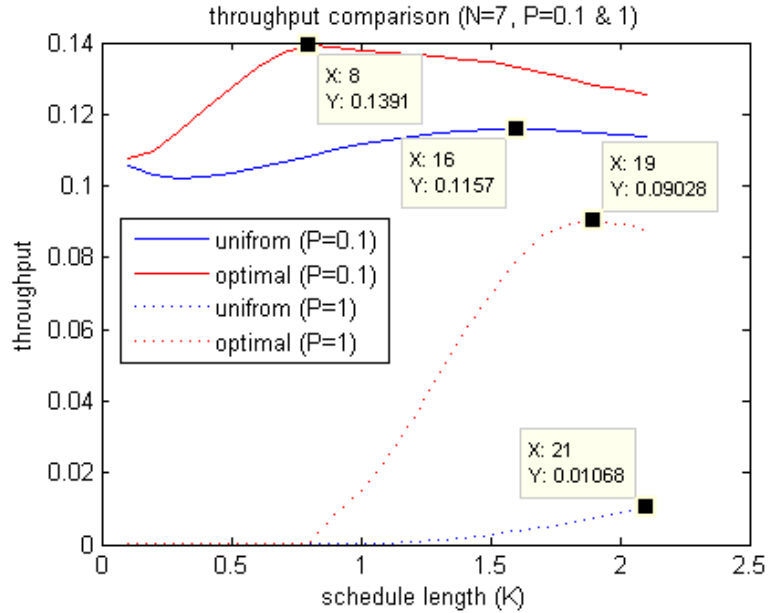

(b)

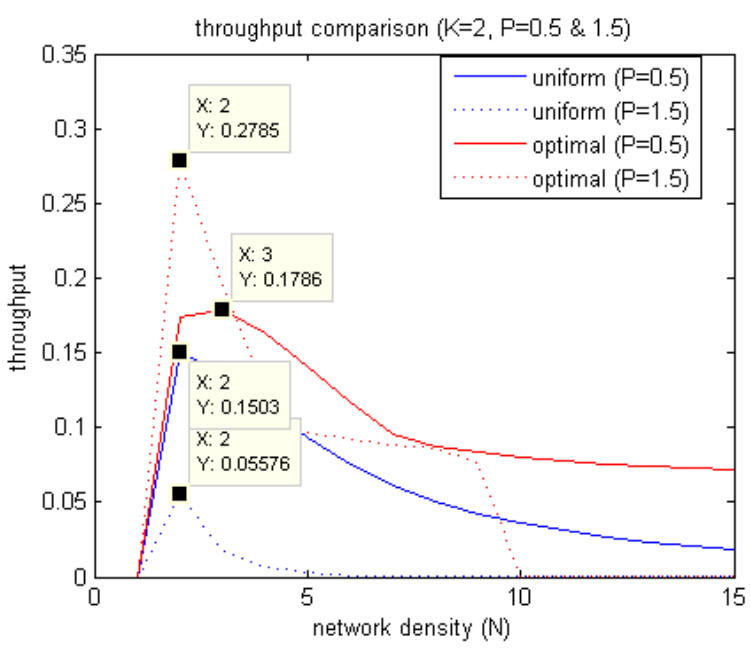

(d)

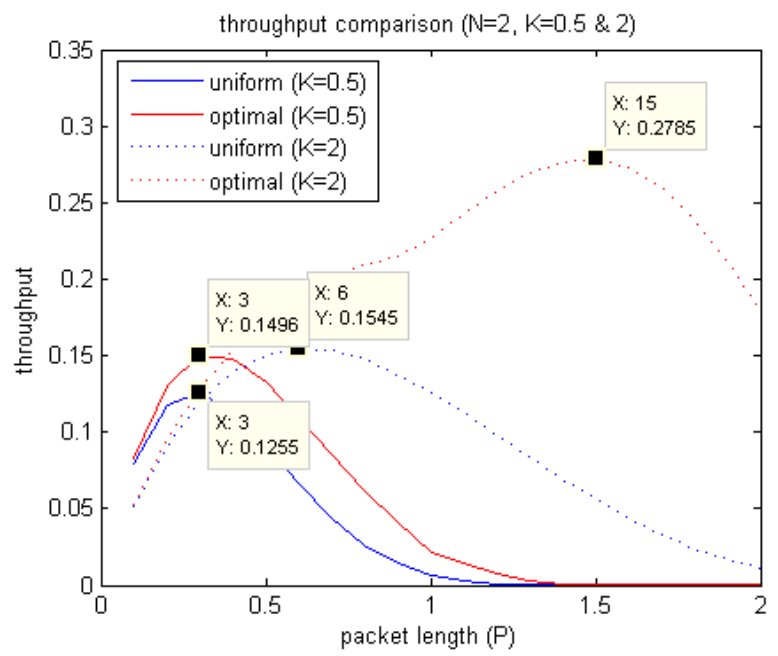

(f)

Fig. 6. Performance evaluation and comparison 


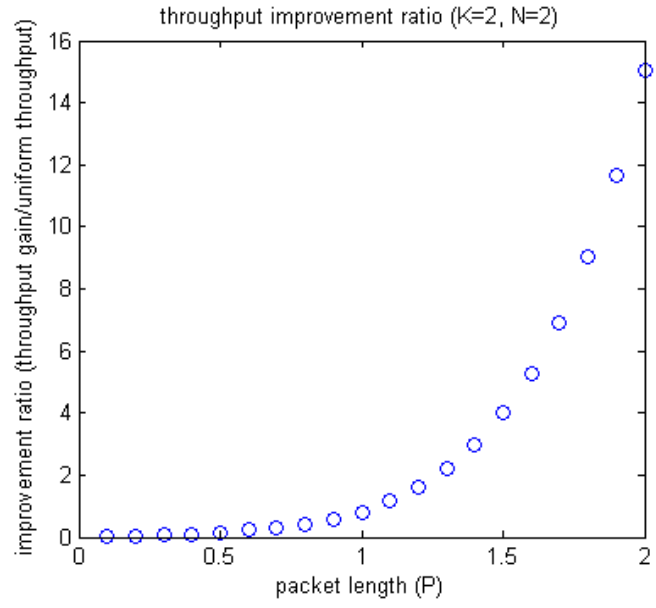

Fig. 7. Throughput improvement ratio vs. packet length $P$

- For optimal DRT scheduler, there exists an throughput optimal packet length and network density, when schedule length is fixed. In general, optimal DRT scheduler achieves higher throughput when density $N$ is low and packet length $P$ is long.

- Both policies benefit from low network density. In other words, DRT strategy-based schedulers are suitable for low contention environments.

\section{CONCLUSIONS}

In this paper, we prove the NP-completeness and hard-toapproximate ratio of Metric Underwater Scheduling. Having seen that collision-free scheduling is intractable, we then focus on low-complexity distributed, randomized, topologyindependent (DRT) schemes. In light of our analysis results, we find that uniform DRT scheduler, which is the throughputoptimal DRT strategy-based scheduler for terrestrial RF networks, performs poorly in UWASN. We find the optimal DRT scheduler by using nonlinear programming, and characterize its properties. The performance comparison of optimal DRT scheduler and uniform DRT scheduler is also presented. Although the propagation delay is the major issue influencing the throughput of UWASN, it is possible to significantly improve throughput without the knowledge of network topology. In general, optimal DRT scheduler performs highest throughput in low contention environments and prefers long packet. We plan to extend this work to a multi-hop network setting. In addition, we are also interested in applying our findings to design a medium access protocol, and a collision resolution protocol.

\section{ACKNOWLEDGMENT}

The work described here is supported in part by NSF through grants CNS-0627028. Any opinions, findings, and conclusions or recommendations expressed in this material are those of the authors and do not necessarily reflect the views of the NSF, NASA, or USC Viterbi School of Engineering.

\section{REFERENCES}

[1] J. Partan, J. Kurose, and B. Levine, "A survey of practical issues in underwater networks," Special section on ACM WUWNet, pp. 23-33, 2007.

[2] I. Akyildiz, D. Pompili, and T. Melodia, "Underwater acoustic sensor networks: research challenges," Elsevier Ad Hoc Networks, vol. 3, pp. 257-279, 2005.

[3] J. Heidemann, W. Ye, J. Wills, A. Syed, and Y. Li, "Research challenges and applications for underwater sensor networking," Proceedings of the IEEE Wireless Communications and Networking Conference, pp. 228235, 2006.

[4] U. Feige and J. Kilian, "Zero knowledge and the chromatic number," $J$. Comput. System Sci., vol. 57, pp. 187-199, 1998.

[5] A. Syed, W. Ye, J. Heidemann, and B. Krishnamachari, "Understanding spatio-temporal uncertainty in medium access with aloha protocols," Proceedings of the second workshop on Underwater networks, pp. 4148, 2007.

[6] J. Ahn and B. Krishnamachari, "Performance of a propagation delay tolerant aloha protocol for underwater wireless networks," Proceedings of IEEE DCOSS, 2008.

[7] M. Molins and M. Stojanovic, "Slotted-fama: a mac protocol for underwater acoustic networks," Proceedings of the IEEE OCEANS, 2006.

[8] H. Nguyen, S. Shin, and S. Park, "State-of-the-art in mac protocols for underwater acoustics sensor networks," Emerging Directions in Embedded and Ubiquitous Computing.

[9] P. Casari, S. Marella, and M. Zorzi, "A comparison of multiple access techniques in clustered underwater acoustic networks," Proceedings of IEEE OCEANS, 2007.

[10] J. Preisig, "Acoustic propagation considerations for underwater acoustic communications network development," Proceedings of ACM WUWNet, 2006.

[11] L. Badia, M. Mastrogiovanni, C. Petrioli, S. Stefanakos, and M. Zorzi, "Analysis and algoritm: an optimization framework for joint sensor deployment, link scheduling and routing in underwater sensor networks," Proceedings of ACM WUWNet, 2006.

[12] J. Kleinberg and E. Tardos, "Algorithm design." Addison Wesley Longman.

[13] http://mathworld.wolfram.com/SquareLinePicking.html, "Square line picking."

[14] P. Huang, Y. Chen, A. Kumar, and B. Krishnamachari, "Link scheduling in a single broadcast domain underwater networks," USC Engineering School Technical Report, 2009. 